\title{
Erratum to: Polymer monolithic column containing embedded graphene oxide sheets for sensitive determination of carbamate insecticides by HPLC
}

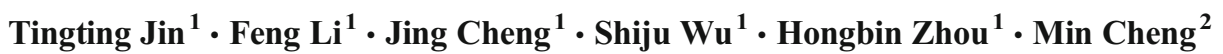

Published online: 21 December 2015

(C) Springer-Verlag Wien 2015

Erratum to: Microchim Acta

DOI 10.1007/s00604-015-1637-y

The original version of this article contained an error. The correct names of the authors are as follows:

Tingting Jin - Feng Li • Jing Cheng - Shiju Wu • Hongbin Zhou $•$ Min Cheng

The online version of the original article can be found at http://dx.doi.org/ 10.1007/s00604-015-1637-y.

Jing Cheng

chengjingok@mail.ccnu.edu.cn

$\triangle \quad$ Min Cheng

494350301@qq.com

1 Key Laboratory of Pesticide and Chemical Biology, Ministry of Education, Institute of Environmental Chemistry, College of

Chemistry, Central China Normal University, Wuhan 430079, China

2 School of Mechanical Science and Engineering, Hua Zhong

University of Science and Technology, Wuhan 430074, China 